Einsetzen in (23) liefert:

$$
\begin{aligned}
I= \pm i \sum_{\alpha, \beta} e_{\alpha} q_{\beta} & \left\{\left\langle\mathfrak{f}\left|D_{\alpha \beta}\right| \mathfrak{f}\right\rangle\right. \\
& \left.+\frac{1}{m}\left\langle\mathfrak{f}\left|p_{\beta}\right| \mathfrak{f}\right\rangle\left[\left\langle\mathfrak{f}\left|p_{\alpha}\right| \mathfrak{f}\right\rangle-\hbar k_{\alpha}\right]\right\} .
\end{aligned}
$$

Der Whitfieldsche Kopplungsfaktor enthält nur das erste Glied mit $D_{\alpha \beta}$; im Sinne einer korrekten Linearisierung kommt dazu, wie man sieht, noch ein weiteres Glied. Allerdings wird dieses Glied, das für freie Elektronen verschwindet, meistens von untergeordneter Bedeutung sein. Es zeigt sich also, daß die Methode des Deformationspotentials im wesentlichen auf eine Linearisierung hinausläuft.

Zusammenfassend kann man sagen, daß die Formulierungen von Sommerfeld und Bethe sowie von Jones höchst fragwürdig sind. Dagegen sind die Kopplungsfaktoren von Nordheim, Bardeen und Whitfield günstige Näherungen, wobei die beiden ersteren auf Metalle zugeschnitten sind, letzterer dagegen vornehmlich für Halbleiter gedacht ist. Die Ausdrücke von Nordheim und Bardeen führen für kleine $|\mathfrak{q}|$ unmittelbar zur ,,isotropen“ Form (25), nicht aber zur allgemeinen Form (24); schuld daran ist, daß dabei die Elektronen durch ebene Wellen beschrieben werden, die an sich für freie Teilchen in einem isotropen Medium gelten. Andererseits gelten die Ausdrücke von Nordheim und Bardeen| in ihrer allgemeinen Form nicht nur für kleine q. Dagegen stehen die Folgerungen, die diese Autoren für U-Prozesse ziehen, im Gegensatz zu unseren Ergebnissen (vgl. Abschn. 3); auch dies liegt an der vereinfachenden Annahme ebener Wellen.

\title{
Flache Elektronen-Traps und IR-Stimulation bei ZnS-Phosphoren
}

\author{
N. Riehl, G. BaUr und L. Mader \\ Physik-Department der Technischen Hochschule München \\ Herrn Professor Dr.-Ing. H. GoBRecht zum 60. Geburtstag gewidmet \\ (Z. Naturforsch. 24 a, 1296-1302 [1969] ; eingegangen am 6. Juni 1969)
}

\begin{abstract}
Aus Glowkurvenmessungen an ZnS-Phosphoren im Temperaturgebiet unterhalb $100^{\circ} \mathrm{K}$ ergibt sich eine ,,effektive Haftstellenverteilung", die sehr stark von den Präparationsbedingungen abhängt. Neben sehr breiten, quasikontinuierlichen Glowmaxima beobachtet man auch scharfe, diskrete Glowpeaks zwischen $60^{\circ} \mathrm{K}$ und $80^{\circ} \mathrm{K}$.

Die Existenz flacher Haftstellen $(<0,1 \mathrm{eV})$ ist notwendig für die Stimulierbarkeit von $\mathrm{ZnS}$ mit langwelligem IR (bis über $25 \mu$ ). Die wichtigste experimentelle Voraussetzung für die Untersuchung solcher Stimulationseffekte ist die Fernhaltung von unerwünschtem Infrarot von wärmeren, ungekühlten Teilen der Apparatur und der Umgebung. Unter diesen Bedingungen gelingt es, die IR-Stimulation bis zu Wellenlänge über $25 \mu$ quantitativ zu untersuchen und mit ihrer Hilfe optische Traptiefen zu bestimmen.
\end{abstract}

Flache Haftstellen, die thermisch bei Temperaturen unterhalb $100^{\circ} \mathrm{K}$ entleert werden können, bestimmen weitgehend das Lumineszenzverhalten von ZnS-Phosphoren bei tiefen Temperaturen. Diese Haftstellen sind nicht nur für die Form der Glowkurve (Thermolumineszenz), sondern auch für das Nachleuchten bei konstant gehaltener tiefer Temperatur, etwa bei $4,2^{\circ} \mathrm{K}$ (,,Tunnelnachleuchten“") verantwortlich ${ }^{1}$ und für die IR-Stimulation ${ }^{2}$.

Sonderdruckanforderungen erbeten an Prof. Dr. N. RIEHL Physik Department der Technischen Hochschule,

D.8000 München 2, Arcisstr. 21.

1 N. Riehl, Intern. Conf. on Luminescence, Budapest 1966. - L. Mader u. N. Riehl, Z. Phys. 206, 319 (1967).

- N. Riehl, G. Baur. L. Mader u. P. Thoma, Intern. Conf. on II-VI Semiconducting Compounds, Providence 1967.
Die Existenz flacher Traps ist eine notwendige Voraussetzung für die Stimulierbarkeit von $\mathrm{ZnS}$ Phosphoren mit langwelligem IR, und je nach der Art der Trapverteilung lassen sich die verschiedenen Phosphore gut, schlecht oder überhaupt nicht mit langwelligem IR stimulieren. - Über unsere neueren Ergebnisse auf diesem Gebiet, insbesondere hinsichtlich der IR-Stimulation, soll im folgenden berichtet werden.

2 G. Baur, J. Kxobloch, N. Riehl u. P. Thoma, Z. Naturforsch. 21 a, 851 (1966). - G. Baur, J. K уовLOCH, N. Riehl u. P. Thoma, Intern. Conf. on Luminescence, Budapest 1966. - G. Baur, N. Riehl u. P. Thoma, Z. Phys. 206, 229 [1967]. 


\section{Ergebnisse von Thermolumineszenz- Messungen}

Mit Hilfe der Thermolumineszenzmethode wurde die Haftstellenverteilung einer großen Zahl verschiedener ZnS-Phosphore untersucht. Zweck dieser Untersuchungen war es, Phosphore zu finden, die eine sehr hohe Konzentration flacher Traps aufweisen, d.h. sich sehr gut mit IR stimulieren lassen.

Bei Thermolumineszenz- oder Glowkurvenmessungen im Tieftemperaturbereich (d.h. unterhalb $100^{\circ} \mathrm{K}$ ) kommt zu den zur Erreichung genauer und reproduzierbarer Ergebnisse notwendigen Voraussetzungen (lineares Heizprogramm, langsame Aufheizung, dünne Leuchtstoffschichten, Anregung bis zur Sättigung) eine weitere sehr wichtige Bedingung hinzu: Die Probe muß sehr sorgfältig gegen störende IR-Strahlung von wärmeren Teilen der Umgebung abgeschirmt werden, um eine Verfälschung der gemessenen Trapverteilung durch IR-Stimulation auszuschalten.

\section{Allgemeines}

In ZnS-Phosphoren findet man prinzipiell zwei verschiedene Sorten von Verteilungen flacher Traps.

Abbildung 1 zeigt je ein Beispiel, und zwar Abb. 1A zunächst einen Phosphor mit einem Kontinuum flacher Traps. Um beurteilen zu können, ob es sich um eine (kontinuierliche) Trapverteilung handelt, wurde die Wartezeit zwischen dem Ende der Anregung und dem Beginn der Aufheizung variiert. Bei sofortiger Aufheizung erhält man nur eine abfallende Glowemissionskurve (Kurve a). Nach einiger Wartezeit bei $4,2^{\circ} \mathrm{K}$ sind dagegen die flachsten Traps bereits geleert, und man beobachtet in der Glowkurve einen breiten Peak, der sich mit zunehmender Wartezeit zu immer höherer Temperatur verschiebt (Kurve b: 8,5 min Abklingzeit, Kurve c: $\mathbf{3 4}$ min Abklingzeit). Es handelt sich hier also nicht um einen echten Glowpeak, sondern um ein Artefakt, das durch das vorherige Abklingen des Phosphors verursacht wird.

Abbildung 1B zeigt einen Phosphor mit einem scharf ausgeprägten Peak um $60^{\circ} \mathrm{K}$. Hier beobachtet man während der Wartezeit lediglich eine Intensitätsabnahme dieses Peaks, aber keine PeakVerschiebung, was auf einen diskreten Trap hindeutet. (Kurve a: ohne vorheriges Abklingen, Kurve b: 40 min Abklingzeit). Wir sehen, daß man
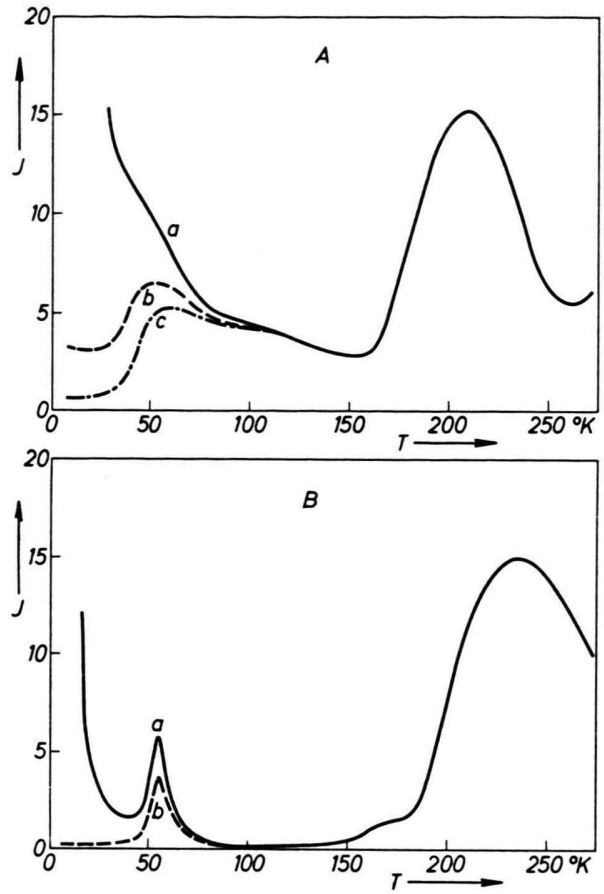

Abb. 1 A. Glowkurven eines $\mathrm{ZnS}(\mathrm{Cu})$-Phosphors mit einem Kontinuum flacher Traps. Kurve $a=0$ Min. Abklingen, Kurve $b=8,5$ Min. Abklingen, Kurve $c=34$ Min. Abklingen.

Abb. 1B. Glowkurven eines $\mathrm{ZnS}(\mathrm{Cu}, \mathrm{Ga})$-Phosphors mit einem Peak bei $60^{\circ} \mathrm{K}$. Kurve $a=0 \mathrm{Min}$. Abklingen, Kurve $b=40$ Min. Abklingen.

bei beiden Phosphoren eine rasche und starke Entleerung flacher und auch tieferer Traps beobachtet, obwohl die Phosphore auf der Temperatur des flüssigen Heliums (ca. $6^{\circ} \mathrm{K}$ ) gehalten werden. Wir konnten auch nachweisen, daß es sich bei dieser Trapentleerung um leuchtende Rekombinationen der befreiten Elektronen mit angeregten Aktivatoren handelt ${ }^{1}$. Bei dieser starken Entleerung flacher und auch verhältnismäßig tiefer Traps (bis zu ca. $0,1 \mathrm{eV}$ ) kann es sich natürlich nicht um eine thermische Befreiung der getrappten Elektronen handeln, sondern es müssen direkte Übergänge der getrappten Elektronen zu den angeregten Aktivatoren erfolgen ${ }^{1,3}$.

\section{Trapkontinua}

Um einen Überblick über die möglichen Verteilungen flacher Traps in $\mathrm{ZnS}$ zu gewinnen, wurde eine große Anzahl von verschiedenen Phosphoren untersucht.

3 N. RIEHL, Festkörperprobleme VIII, 232 (1968). 
Abbildung 2 zeigt vier Proben von $\mathrm{ZnS}$-Phosphoren mit verschiedenen kontinuierlichen Verteilungen flacher Traps. Wir sehen eine Probe praktisch ohne flache Traps und drei weitere mit steigendem Trapkontinuum (bezogen auf den $160^{\circ} \mathrm{K}$-Peak, der bei allen etwa gleich stark ausgeprägt ist).

Obwohl diese Proben praktisch gleich hergestellt sind, ist das Kontinuum der flachen Traps sehr verschieden, während die tieferen, für den $160^{\circ} \mathrm{K}$-Peak verantwortlichen Traps bei den verschiedenen Proben praktisch gleich sind. Die starken Unterschiede bei den flachen Traps sind auf geringe, unbeabsichtigte und unbekannte Abweichungen in den Herstellungsbedingungen zurückzuführen, die man nicht unter Kontrolle hat.

Es ist sehr wahrscheinlich, daß die sog. Koaktivatoren und dabei vor allem das Chlor, aber auch der Sauerstoff bei der Bildung der flachen Traps eine gewisse Rolle spielen. Die leichte Beeinflußbarkeit der flachen Traps spricht jedoch dafür, daß es hier nicht nur auf ,chemische Verunreinigungen", d.h. den Einbau bzw. eine indirekte Wirkung von Fremdatomen ankommt, sondern auch auf ,,physikalische Defekte“, wie z.B. eine Fehlordnung im Kristallgitter. Weiterhin könnte auch die Tatsache, daß in ZnS-Phosphoren Wurtzit- und BlendeStruktur meist nebeneinander im gleichen Kristall vorliegen, mit verantwortlich sein für die Bildung und Verteilung flacher Traps.

Bisher haben wir immer gleiche Präparation vorausgesetzt und bereits sehr verschiedene Trapverteilungen gefunden. Variiert man jetzt noch die Präparationsbedingungen (z. B. Ausgangsmaterial, Glühgas, Glühtemperatur und Glühdauer sowie $\mathrm{Zu}$ sätze zum Glühgut), so erhöht sich die Vielfalt der Verteilungen flacher Traps in $\mathrm{ZnS}$ noch beträchtlich und man kann durch geeignete Wahl der Herstellungsbedingungen praktisch jede beliebige Trapverteilung erhalten. Ergänzend zu den in Abb. 2 gezeigten Glowkurven gibt Abb. 3 noch vier Beispiele für die Vielfalt der möglichen Trapverteilungen in $\mathrm{ZnS}$.

Interessant ist, daß bei gleich präparierten Phosphoren die Art des Aktivators keinen wesentlichen
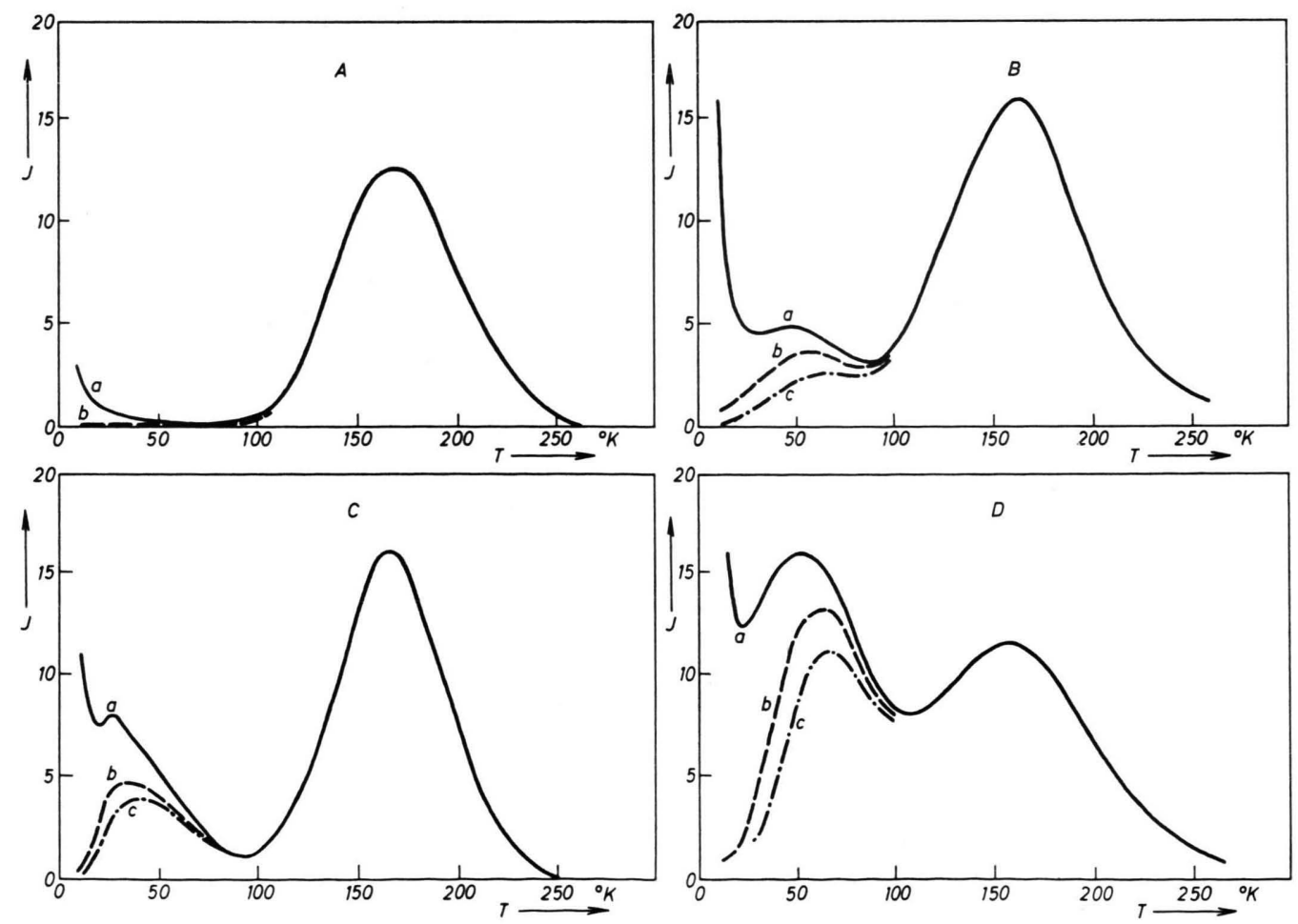

Abb. 2 A: Glowkurven eines ZnS-Phosphors, der fast keine flachen Traps hat.

Abb. 2B-D. Glowkurven von ZnS-Phosphoren mit steigendem Kontinuum flacher Traps (bezogen auf den $160^{\circ} \mathrm{K}$-Peak). Kurve $a=$ jeweils 0 Min. Abklingen,

Kurve $b=$ jeweils ca. 10 Min. Abklingen, Kurve $c=$ jeweils ca. 40 Min. Abklingen. 

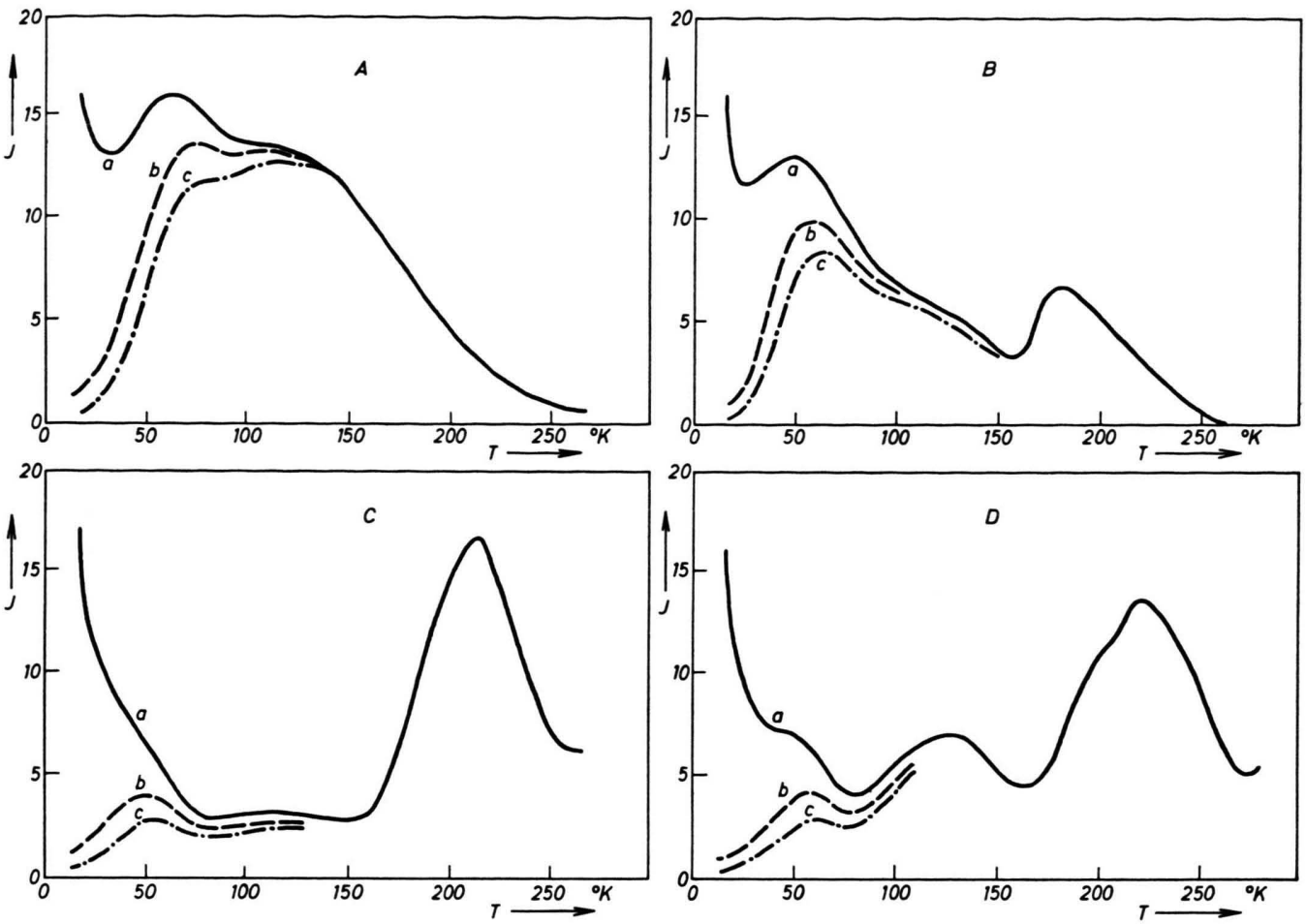

Abb. 3A-D. Glowkurven von vier ZnS-Phosphoren verschiedener Herstellung mit sehr unterschiedlicher Trapverteilung (Bezeichnung der Kurven $a, b, c$ siehe Abb. 2).
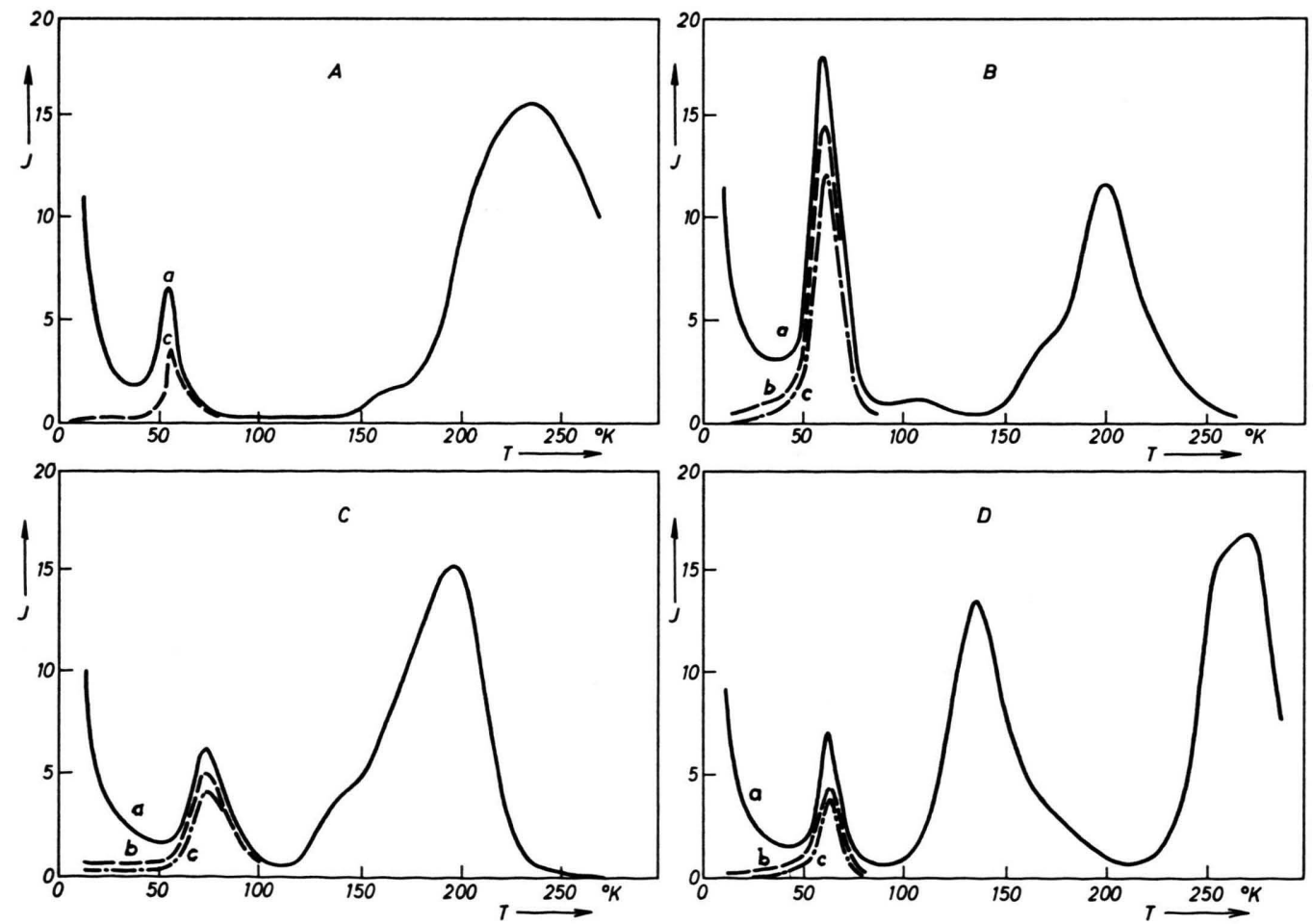

Abb. 4A-B: Glowkurven von $\mathrm{ZnS}(\mathrm{Cu}, \mathrm{Ga})$-Phosphoren verschiedener Herkunft. 4C: Glowkurven eines $\mathrm{ZnS}(\mathrm{Cu}, \mathrm{In})$ Phosphors. 4D: Glowkurven eines rotleuchtenden $\mathrm{ZnS}(\mathrm{Cu})$-Phosphors (Bezeichnung der Kurven $a, b, c$ siehe Abb. 2). 
Einfluß auf die Trapverteilung hat. Wir konnten sowohl bei aktivatorfreiem $\mathrm{ZnS}$ als auch bei $\mathrm{Cu}$ oder Ag-aktivierten ZnS-Phosphoren gleicher Präparation jeweils gleiche Trapverteilungen beobachten. Dagegen kann die Aktivatorkonzentration wohl eine Rolle spielen.

\section{Diskrete Traps}

Neben kontinuierlichen Trapverteilungen können in $\mathrm{ZnS}$ im Temperaturbereich unterhalb $100^{\circ} \mathrm{K}$ auch scharfe diskrete Peaks auftreten. Abbildung 4 zeigt mehrere ZnS-Phosphore mit einem diskreten Trap im Temperaturbereich von $60^{\circ} \mathrm{K}$ bis $80^{\circ} \mathrm{K}$. Hier handelt es sich um folgende Phosphore: In $\mathrm{Abb} .4 \mathrm{~A}$ und $4 \mathrm{~B}$ um $\mathrm{ZnS}(\mathrm{Cu}, \mathrm{Ga})$-Phosphore verschiedener Herkunft, in Abb. $4 \mathrm{C}$ um $\mathrm{ZnS}(\mathrm{Cu}, \mathrm{In})$ und in $\mathrm{Abb} .4 \mathrm{D}$ um einen $\mathrm{ZnS}(\mathrm{Cu})$-Phosphor ohne Koaktivator.

Allen diesen Proben ist gemeinsam, daß sie unter Ausschluß von Sauerstoff unter $\mathrm{H}_{2} \mathrm{~S}$-Atmosphäre durch 1- bis 2 -stündiges Glühen bei 1100 bis $1200^{\circ} \mathrm{C}$ hergestellt sind. Alle Proben mit einem solchen diskreten Peak unterhalb $100^{\circ} \mathrm{K}$ zeigen - zumindest bei tiefen Temperaturen - rote Lumineszenz. Dabei kann es sich um Cu-aktivierte oder aktivatorfreie Phosphore handeln. Die Anwesenheit, die Art und die Konzentration der dreiwertigen Koaktivatoren scheint nur auf die Temperaturlage dieses Peaks Einfluß zu haben, nicht aber auf sein Auftreten überhaupt.

$\mathrm{Da} B$ diese Peaks wirklich mit der roten Lumineszenz von $\mathrm{ZnS}$ in Zusammenhang stehen, sieht man auch daran, daß sie verschwinden, wenn man solche rotleuchtende ZnS-Phosphore unter Zusatz von Chlorid oder in einer HCl-Atmosphäre nachglüht. Man erhält dann übliche grün oder blau leuchtende ZnS-Phosphore, auch wenn dreiwertige „Koaktivatoren“ vorhanden sind.

\section{IR-Stimulation}

Stimulationsexperimente bei und oberhalb der Temperatur des flüssigen Stickstoffs wurden insbesondere von Urbach, von Kallmann und Mitarbeitern sowie von Broser und Mitarbeitern durchgeführt. Einen guten Uberblick über alle bis 1965 erschienenen Arbeiten gab MASON ${ }^{4}$. Die obere Grenze der Stimulierbarkeit lag bei allen Autoren

4 J. Mason, Rev. Mod. Phys. 37, 743 (1965). in der Gegend von 2,5 $\mu$ bei der Temperatur des flüssigen Stickstoffs.

Wir haben diese Experimente auf den Tieftemperaturbereich (zwischen $4,5^{\circ} \mathrm{K}$ und $77^{\circ} \mathrm{K}$ ) ausgedehnt mit dem Ziel, die Stimulierbarkeit von Phosphoren mit langwelligem IR $\mathrm{zu}$ untersuchen. Man muß dabei sorgfältig darauf achten, daß jegliche IR-Störstrahlung von ungekühlten Teilen der Apparatur (sie wirken als thermische Strahler) zurückgehalten wird. Dazu ist eine Abschirmung auf der Temperatur des flüssigen Heliums erforderlich, deren optische Eingänge IR um mindestens einen Faktor $10^{10}$ schwächen. Die Forderungen, die an eine für diese Zwecke geeignete Apparatur gestellt werden müssen, sind:

a) Die Probe darf keine Teile der Apparatur sehen, die wärmer als $10^{\circ} \mathrm{K}$ sind.

b) Verschlüsse und optische Eingänge müssen fremdes IR mindestens um einen Faktor $10^{10}$ schwächen.

c) Will man monochromatisches IR auf die Probe einstrahlen, so sind ein gekühlter Monochromator oder gekühlte Filter erforderlich.

Wir haben diese experimentellen Probleme auf verschiedene Arten gelöst. Abbildung 5 zeigt einen Querschnitt durch eine unserer Apparaturen. Mit dieser Apparatur erhielten wir folgende experimentellen Ergebnisse:

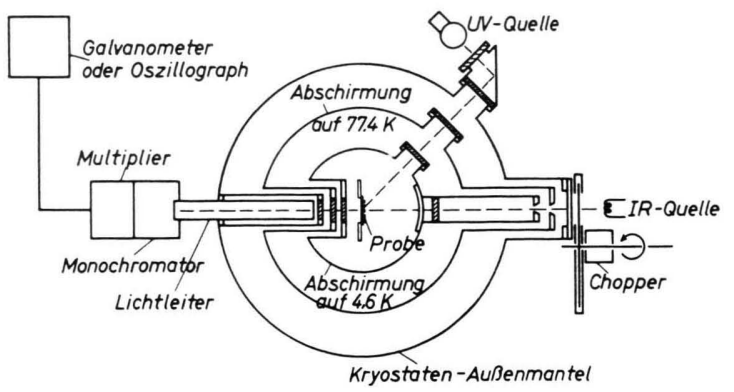

Abb. 5. Schematische Darstellung der Meßanordnung.

1. Die Grenzwellenlänge der Stimulation liegt über $20 \mu$. Die obere Grenze der Stimulation können wir noch nicht genau angeben, da uns entsprechende Filter nicht zur Verfügung stehen. Wir haben aber gut meßbare Effekte mit IR-Wellenlängen zwischen 20 und $25 \mu$ gefunden.

2. Der zeitliche Anstieg der stimulierten Emission nach dem Beginn der IR-Einstrahlung ist steiler als 1 msec. Auf 600 Hertz IR-Rechteckimpulse antwortet der Phosphor mit den entsprechenden 


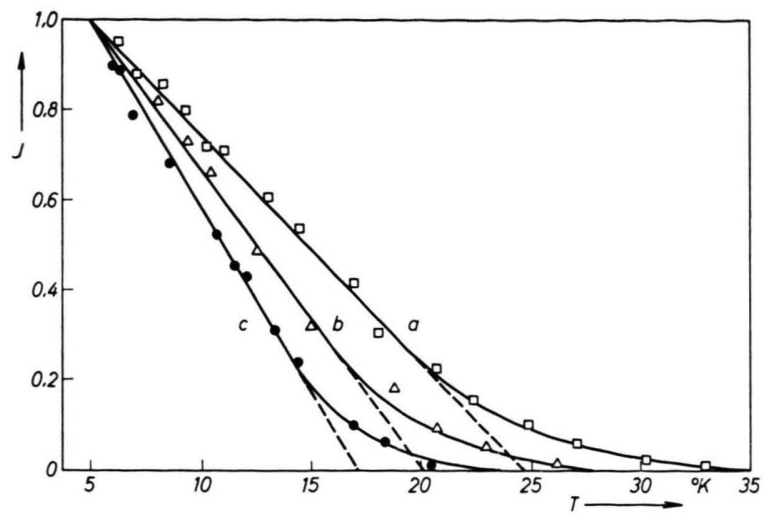

Abb. 7. Stimulationsausbeute eines blau leuchtenden $\mathrm{ZnS}(\mathrm{Cu})$-Phosphors in Abhängigkeit von der Temperatur. Kurve $a=$ Stimulation mit IR: $9,6 \mu$, Kurve $b=$ Stimulation mit IR: $14,5 \mu$, Kurve $c=$ Stimulation mit IR : 20 bis $25 \mu$. größer wird. In Tab. 1 sind die so gewonnenen optischen Traptiefen und die aus Thermolumineszenzmessungen erhaltenen Werte zusammengestellt.

\begin{tabular}{llll}
\hline IR $\mu$ & 9,6 & 14,5 & 20 \\
\hline$E_{\text {opt }} \mathrm{eV}$ & 0,13 & 0,085 & 0,062 \\
$E_{\text {th }} \mathrm{eV}$ & 0,038 & 0,033 & 0,028 \\
\hline
\end{tabular}

Tab. 1.

Ein Teil der vorliegenden Meßergebnisse stammt aus den Diplomarbeiten der Herren Besigk, H.-P. Braun, A. Gierl und H. Selzle. - Diese Untersuchungen wurden durch die Fraunhofer-Gesellschaft gefördert.

\title{
Diffusion von $\mathrm{In}$ und $\mathrm{Cu}$ in $\mathrm{ZnS}$-Einkristallen
}

\author{
H. Nelkowski und G. Bollmann
}

II. Physikalisches Institut der Technischen Universität Berlin

Herrn Professor Dr.-Ing. H. GoвREснт zum 60. Geburtstag gewidmet

(Z. Naturforsch. 24 a, 1302-1306 [1969] ; eingegangen am 16. Juni 1968)

The diffusion of $\mathrm{In}$ and $\mathrm{Cu}$ in $\mathrm{ZnS}$ single crystals was investigated by means of radioactive tracers. The diffusion constant $D_{0}$ and the activation energy $E_{\mathrm{A}}$ of the diffusion equation $D=$ $D_{0} \cdot \exp \left(-E_{\mathrm{A}} / k T\right)$ were determined by analysing the temperature dependence of the concentration profiles. The result for $\operatorname{In}\left(E_{\mathrm{A}}=2,2 \mathrm{eV}, D_{0}=30 \mathrm{~cm}^{2} \mathrm{~s}^{-1}\right)$ is interpreted as a diffusion via $\mathrm{Zn}$ vacancies and that for $\mathrm{Cu}\left(E_{\mathrm{A}}=0,79 \mathrm{eV}, D_{0}=2,6 \cdot 10^{-3} \mathrm{~cm}^{2} \mathrm{~s}^{-1}\right)$ as a diffusion via interstitial sites which probably is influenced by $\mathrm{Zn}$ vacancies. - Preceding doping with In significantly retards the $\mathrm{Cu}$ diffusion, consistent with the model of $\mathrm{Cu}$-In pair formation. The reasons for some deviations of the experimental data from the calculated concentration profiles are discussed. - Diffusion measurements with high $\mathrm{Cu}$-concentrations yield a Cu-solubility of $300 \mathrm{ppm}$ at $840 \mathrm{~K}$ and $1000 \mathrm{ppm}$ at $950 \mathrm{~K}$.

Aven und Halsted ${ }^{1}$ haben eingehend die Diffusion von $\mathrm{Cu}$ in $\mathrm{ZnSe}$ sowie deren Wirkung auf die optischen und elektrischen Eigenschaften untersucht. Aus der Temperaturabhängigkeit der Diffusionsprofile erhalten sie für nicht dotierte Kristalle eine Diffusionskonstante von $1,7 \cdot 10^{-5} \mathrm{~cm}^{2} \mathrm{~s}^{-1}$ und eine Aktivierungsenergie von $0,56 \mathrm{eV}$. Eine vorherige Dotierung mit Al bewirkte eine starke Herabsetzung der Cu-Diffusion, was der Bildung von $\mathrm{Cu}$-Al-Komplexen zugeschrieben wird. Wesentlich weniger Informationen liegen über die Diffusion von Störstellen in $\mathrm{ZnS}$ vor. Aven und Halsted geben in der gleichen Arbeit lediglich den Diffusionskoeffi-

Sonderdruckanforderungen erbeten an Dr. H. NELkowski, II. Physikalisches Institut der Technischen Universität Berlin, D-1000 Berlin 12, Hardenbergstr. 34 . zienten für $\mathrm{Cu}$ in $\mathrm{ZnS}$ bei $770 \mathrm{~K}$ zu $1,5 \cdot 10^{-9} \mathrm{~cm}^{2} \mathrm{~s}^{-1}$ an. Im übrigen beschränken sie sich auf die Aussage, daß die Diffusionsvorgänge denen im $\mathrm{ZnSe}$ sehr ähnlich sind. Deshalb haben wir mit radioaktiv markiertem $\mathrm{Cu}$ und In die Diffusion dieser für das Lumineszenzverhalten des $\mathrm{ZnS}$ wichtigen Störstellen im Temperaturgebiet von 750 bis $1250 \mathrm{~K}$ untersucht.

\section{Experimentelle Methode}

Die verwendeten $\mathrm{ZnS}$-Einkristalle waren aus der Schmelze gezogen ${ }^{2}$ und Röntgen-Strukturanalysen ergaben, daß sie eine kubische Struktur mit starker eindimensionaler Fehl-

1 M. Aven u. R. E. Halsted, Phys. Rev. 137, A 228 [1965].

2 Hergestellt von der Firma ,,The Eagle Picher Company“, Miami, Okla., USA. 